\title{
Efeitos de dois tipos de treinamento de natação sobre a adiposidade e o perfil lipídico de ratos obesos exógenos
}

\author{
Effects of two different types of swimming \\ exercise on adiposity and lipid profile \\ in rats with exogenous obesity
}

\author{
Lucimara ZAMBON ${ }^{1}$ \\ Fernanda Oliveira DUARTE2,3 \\ Laura Franco de FREITAS ${ }^{3}$ \\ Flavia Regina Rodrigues SCARMAGNANI ${ }^{3}$ \\ Ana DÂMASO 2,4 \\ Ana Cláudia Garcia de Oliveira DUARTE2,3 \\ Marcela SENE-FIORESE 2,3,5
}

\section{RE S U M O}

\section{Objetivo}

Investigar os efeitos do exercício de natação intermitente em relação ao exercício contínuo e ao sedentarismo, em ratos Wistar, após o desenvolvimento de obesidade exógena pela administração de uma dieta hiperlipídica palatável sobre: evolução do peso corporal, ingestão alimentar, adiposidade, percentual de gordura dos tecidos e perfil lipídico.

\section{Métodos}

Foram utilizados ratos adultos, mantidos em gaiolas individuais, com livre acesso a água e comida. O protocolo experimental incluiu: 1) desenvolvimento da obesidade exógena (3 semanas), os animais foram divididos em: $\mathrm{P}$ : sedentários alimentados com dieta padrão $\operatorname{Primor}^{\circledR}(\mathrm{n}=8)$ e H: sedentários alimentados com dieta hiperlipídica $(n=32) ; 2)$ tratamentos (8 semanas subseqüentes), os animais ( $n=24)$ passaram a receber dieta padrão e foram divididos em: sedentário; treinado contínuo e treinado intermitente. Treinamentos (5x semana): Contínuo (90

$\overline{1}$ Universidade Federal de São Carlos, Programa de Especialização em Fisiologia do Exercício. São Carlos, SP, Brasil.

2 Universidade Federal de São Carlos, Programa de Pós-Graduação em Ciências Fisiológicas. Rod. Washington Luís (SP-310), km 235, 13565-905, São Carlos, SP, Brasil. Correspondência para/Correspondence to: M. SENE-FIORESE. E-mail: $<$ m_sene2004@yahoo.com.br>

${ }^{3}$ Universidade Federal de São Carlos, Departamento de Educação Física e Motricidade Humana, Laboratório de Nutrição e Metabolismo Aplicados ao Exercício. São Carlos, SP, Brasil.

${ }^{4}$ Universidade Federal de São Paulo, Departamento de Ciências da Saúde. Santos, SP, Brasil.

5 Universidade Camilo Castelo Branco, Faculdade de Educação Física. Descalvado, SP, Brasil. 
708 | L. ZAMBOM et al.

minutos/dia) e Intermitente (3×30min/dia). Os animais foram submetidos à eutanásia (3 e 8 semanas), sendo coletados os tecidos adiposos, o fígado e o sangue. Foram determinados a adiposidade e o percentual de gordura dos tecidos adiposos e do fígado, o ganho de peso corporal, o consumo alimentar e o perfil lipídico.

\section{Resultados}

A dieta hiperlipídica aumentou a adiposidade, o percentual de gordura acumulada no fígado e desenvolveu dislipidemias. A troca de dieta e os dois tipos de treinamento foram capazes de reverter o quadro de obesidade exógena. Contudo, o exercício intermitente foi mais eficiente na redução da adiposidade e de ganho de peso.

\section{Conclusão}

Associados à dieta balanceada, os treinamentos aplicados neste estudo poderiam ser utilizados como estratégia no controle de peso e de dislipidemias, tanto em modelos experimentais quanto em seres humanos.

Termos de indexação: Adiposidade. Atividade física. Dislipidemias. Obesidade.

\section{A B S T R A C T}

\section{Objective}

The objective was to look into the effects of intermittent swimming against continuous exercise and inactivity in Wistar rats after they developed exogenous obesity though the consumption of a palatable fat-rich diet. The following was investigated: evolution of body weight, food intake, adiposity, fat percentage in tissues and lipid profile.

\section{Methods}

Adult rats were kept in individual cages with free access to food and water. The experimental protocol included: 1) development of exogenous obesity (3 weeks). The animals were divided into $S$ - inactive animals fed the standard Primor ${ }^{\circledR}$ diet ( $\left.n=8\right)$ and HF - inactive animals fed a high-fat diet $(n=32)$; 2) Treatment (the 8 following weeks) - the animals $(n=24)$ were given the standard diet and divided into an inactive group, a continuous training group and an intermittent training group. Training occurred 5 times per week and was either continuous (90 minutes per day) or intermittent (3 times 30 minutes per day). The animals were killed at 3 and 8 weeks and the fat tissues, the liver and the blood were collected. Adiposity, fat percentage of the fat tissues and liver, gain of body weight, food consumption and lipid profile were determined.

\section{Results}

The high-fat diet increased adiposity, percentage of fat in the liver, and induced dyslipidemias. Diet change and the two types of training were capable of reducing exogenous obesity. However, intermittent exercise was more efficient in reducing adiposity and preventing weight gain.

\section{Conclusion}

When associated with a balanced diet, the trainings used in this study can be used as a strategy to control weight and dyslipidemias, both in experimental models and in human beings.

Indexing terms: Adiposity. Physical activity. Dyslipidemias. Obesity.

\section{N T R O D U Ç Ã O}

A obesidade tem se tornado um problema de saúde pública e está fortemente associada a diversas doenças crônicas não transmissíveis, entre elas: hiperlipidemia, hipertensão arterial, resistência insulínica, dislipidemias, diabetes mellitus, entre outras ${ }^{1,2}$. A maior parte dessas adversidades ou doenças está, particularmente, relacionada com o aumento da adiposidade visceral ${ }^{3,4}$, sendo que as principais causas desse acúmulo são a ingestão excessiva de gordura e o sedentarismo ${ }^{5,6}$. No sentido de minimizar esses efeitos deletérios, a prática de atividade física tem se tornado parte importante nas estratégias não farmacológicas de controle da obesidade e do ganho de peso ${ }^{6}$.

Diante disso, o Colégio Americano de Medicina Esportiva (ACSM) e o Centro para Controle e Prevenção de Doenças (CDC) estipularam que todo adulto sedentário deve: "acumular 30 minutos ou mais de atividade física moderada durante 
o decorrer do dia, todos os dias da semana, para a promoção de saúde e a prevenção de doenças" 7-9. No entanto outros estudos reportam que essa recomendação seria insuficiente para prevenir o ganho de peso e sugerem o acúmulo de 60 minutos de atividade física moderada ${ }^{10}$.

Estudos experimentais têm demonstrado que o exercício contínuo de natação (90 minutos/dia), associado à dieta balanceada, resulta na redução da adiposidade e na melhora do perfil lipídico em ratos ${ }^{11-13}$. Dessa forma, torna-se importante investigar se dividir essa duração (90 minutos/dia), em sessões de 30 minutos, teria o mesmo efeito sobre o ganho de peso, a adiposidade e o perfil lipídico de ratos.

\section{MÉTODOS}

Foram utilizados 40 ratos machos adultos da Linhagem Wistar, com 90 dias de vida, procedentes do Biotério Central da Universidade Federal de São Carlos. Durante todo o período experimental de 11 semanas, os animais foram mantidos em gaiolas individuais no biotério do Laboratório de Nutrição e Metabolismo, do Departamento de Educação Física e Motricidade Humana, com temperatura constante ao redor de $23^{\circ} \mathrm{C}$ (como variação de $1^{\circ} \mathrm{C}$ ) e foto período artificial de 12/12 horas, com livre acesso à água e ao alimento. A manipulação dos animais estava em conformidade com a legislação vigente sobre o assunto no Brasil ${ }^{14}$. O projeto foi aprovado pelo Comitê de Ética em Pesquisa de Experimentação Animal da Universidade Federal de São Carlos (CCEA/UFSCar) (protocolo no 015/2007).

O protocolo experimental foi realizado em duas etapas:

1) Desenvolvimento da obesidade exógena com duração de três semanas, sendo os animais divididos aleatoriamente em dois grupos: sedentários alimentados com dieta padrão $(P, n=8)$ e sedentários alimentados com dieta hiperlipídica $(H, n=32)$. Ao final das três semanas os animais do grupo $\mathrm{P}$ e oito animais do grupo $\mathrm{H}$, selecionados aleatoriamente, foram eutanaziados.
2) Esta fase de tratamento teve duração de oito semanas a partir dos quais os demais animais $(n=24)$ passaram a receber dieta padrão comercial e foram divididos aleatoriamente em três grupos: Sedentário (S); Treinado Contínuo (TC) e Treinado Intermitente (TI).

A dieta padrão utilizada consistiu de ração comercial para ratos, da marca Primor ${ }^{\oplus}$. De acordo com o fabricante, $100 \mathrm{~g}$ dessa dieta contém: $23 \%$ de proteína, $49 \%$ de carboidrato, $4 \%$ de lipídios totais, $5 \%$ de fibras, $7 \%$ de cinzas, $6 \%$ de vitaminas, totalizando $4,07 \mathrm{kcal} / \mathrm{g}$. Já a dieta hiperlipídica foi constituída de mistura de alimentos hiperenergéticos seguindo a proporção: $15 \mathrm{~g}$ de ração padrão, $10 \mathrm{~g}$ de amendoim torrado, $10 \mathrm{~g}$ de chocolate ao leite e $5 \mathrm{~g}$ de biscoito de amido de milho. Estes ingredientes foram triturados e misturados, formando pellets, ofertados aos animais. A dieta hiperlipídica foi previamente padronizada e analisada nos laboratórios do grupo de pesquisa possuindo a cada $100 \mathrm{~g}$ de peso: $20 \%$ de proteína, $48 \%$ de carboidrato, $20 \%$ de lipídios totais, $4 \%$ de fibra, $5 \%$ de vitaminas e minerais, totalizando $5,12 \mathrm{kcal} / \mathrm{g}^{11,12}$.

Foram realizados dois tipos de treinamento moderado de natação. No treinamento contínuo os animais nadaram durante 90 minutos, enquanto que os animais do grupo treinado intermitente nadaram em três sessões de 30 minutos com intervalo de 4 horas entre as sessões, ambos cinco vezes por semana. O treinamento foi realizado em tanques individuais com temperatura controlada entre $30^{\circ} \mathrm{C}$ (com variação de $2^{\circ} \mathrm{C}$ ) e com sobrecarga adicional de 3 a $5 \%$ do peso corporal atado a cauda.

O consumo alimentar e o peso corporal dos animais foram mensurados diariamente, durante todo o período experimental. Para o cálculo do delta do peso corporal (\%) foi usada a fórmula:

$\Delta=[($ Peso final-Peso inicial)/Peso inicial $] \times 100$.

Ao final de cada período experimental (3 e 8 semanas), os animais foram eutanaziados em guilhotina e os tecidos adiposos brancos Epididimal (EPI), Retroperitoneal (RET), Visceral (VIS) e o 
fígado foram retirados e estocados em freezer a $-20^{\circ} \mathrm{C}$ para análises futuras. O sangue foi coletado em tubos heparinizados sendo centrifugado e armazenado para determinação das concentrações de colesterol total, Lipoproteínas de Alta Densidade (HDL-C) e Triacilglicerol (TG). Estes parâmetros foram determinados pelo método enzimático colorimétrico utilizando kits da marca Laborlab $^{\circledast}$, com leitura realizada em espectrofotômetro modelo UV-1601PC (Shimadzu Corp, Kyoto, Japan).

Para a determinação do percentual de gordura dos tecidos foram realizadas a saponificação e a extração dos lipídios pelo método gravimétrico previamente padronizado ${ }^{15}$.

Os dados estão expressos como Média (M) e Desvio-Padrão (DP). Os valores foram avaliados estatisticamente por análise de variância (ANOVA) e foi aplicado o teste de Tukey-Kramer para verificação da existência de diferenças estatísticas entre as médias, com nível de significância de 0,05 para as variáveis analisadas. Foi utilizado o software Instat 3,0 for Windows 95 (GraphPad, San Diego, CA, USA, 1998).

\section{RESULTADOSE DISCUSSÃO}

Diversos estudos têm discutido os efeitos do consumo de dietas hiperlipídicas sobre a adiposidade intra-abdominal e a sua relação com o desenvolvimento de obesidade e doenças crônicas não transmissíveis ${ }^{16-19}$. Os efeitos dessas dietas sobre o peso corporal e o consumo alimentar apresentam resultados controversos na literatura, principalmente devido ao tempo de administração ${ }^{11,12,20-22}$.

Na primeira fase do presente estudo, a redução no consumo alimentar expresso em gramas (g) dos animais alimentados com dieta hiperlipídica parece estar relacionada ao aumento na quantidade de energia presente nesse tipo de dieta $(5,12 \mathrm{kcal} / \mathrm{g})$, quando comparado à dieta padrão (P) $(4,07 \mathrm{kcal} / \mathrm{g})$ (Tabela 1$)^{23}$. Dietas ricas em gordura reduzem a eficiência alimentar ${ }^{13} \mathrm{e}$ aumentam a eficiência metabólica, o que pode explicar porque a dieta hiperlipídica não apresentou resultados estatisticamente significantes sobre o ganho de peso (Tabela 1).

Apesar disso, os animais alimentados com dieta hiperlipídica apresentaram aumento no peso relativo dos tecidos adiposos brancos epididimal, retroperitoneal e visceral, indicando aumento na adiposidade central e visceral (Tabela 2), como demonstrado previamente ${ }^{12,20-22,24}$. Além disso, também houve aumento no percentual de gordura desses tecidos, quando comparado com o grupo alimentado com dieta padrão. Ressalta-se que,

Tabela 1. Peso inicial e final, ganho de peso, ingestão energética diária e consumo alimentar diário de grupos experimentais (ratos Wistar). São Carlos (SP), 2007.

\begin{tabular}{|c|c|c|c|c|c|c|c|c|c|c|c|}
\hline \multirow{3}{*}{ Grupos } & & \multicolumn{4}{|c|}{ Peso (g) } & \multirow{2}{*}{\multicolumn{2}{|c|}{$\begin{array}{c}\text { Ganho de peso } \\
(\%)\end{array}$}} & \multirow{2}{*}{\multicolumn{2}{|c|}{$\begin{array}{l}\text { Ingestão energética } \\
\text { diária (kcal) }\end{array}$}} & \multirow{2}{*}{\multicolumn{2}{|c|}{$\begin{array}{c}\text { Consumo alimentar } \\
\text { diário }(\mathrm{g})\end{array}$}} \\
\hline & & \multicolumn{2}{|c|}{ Inicial } & \multicolumn{2}{|c|}{ Final } & & & & & & \\
\hline & & $\mathrm{M}$ & DP & $\mathrm{M}$ & DP & M & DP & M & DP & M & DP \\
\hline \multirow{2}{*}{ 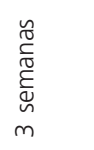 } & P & 225,63 & 6,86 & 329,69 & 11,24 & 46,22 & 6,10 & 107,08 & 5,99 & 26,31 & 1,47 \\
\hline & $\mathrm{H}$ & 225,50 & 9,35 & 339,39 & 20,54 & 50,54 & 7,51 & 122,10 & $9,91^{*}$ & 23,85 & $1,93^{*}$ \\
\hline \multirow{3}{*}{ 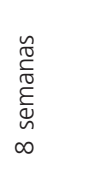 } & $S$ & 352,00 & 18,17 & 454,28 & 16,38 & 29,18 & 3,81 & 96,73 & 4,27 & 23,77 & 1,05 \\
\hline & $\mathrm{TC}$ & 346,66 & 18,98 & 453,83 & 26,36 & 31,19 & 9,60 & 112,35 & $9,53^{+}$ & 27,60 & $2,34^{+}$ \\
\hline & $\mathrm{Tl}$ & 339,50 & 21,64 & 401,25 & $16,03^{+\#}$ & 19,74 & $6,03^{+\#}$ & 115,38 & $4,83^{+}$ & 28,35 & $1,19^{+}$ \\
\hline
\end{tabular}

Diferentes subscritos * versus $\mathrm{P}$; ${ }^{\circ}$ versus $\mathrm{H}_{\text {; }}{ }^{+}$versus $\mathrm{S}$; \# $\mathrm{TC} \times \mathrm{TI} p<0,05$.

P: animais sedentários alimentados com dieta padrão durante 3 semanas; $\mathrm{H}$ : animais sedentários alimentados com dieta hiperlipídica durante

3 semanas; S: sedentário; TC: treinado contínuo; TI: treinado intermitente; M: média; DP: desvio-padrão. 
neste estudo, a adiposidade central refere-se ao acúmulo de tecido adiposo retroperitoneal e a adiposidade visceral ao acúmulo dos tecidos adiposos epididimal e visceral.

O fígado é o principal regulador do fluxo metabólico corporal, recebendo entradas do intestino através do sistema porta-hepático, da circulação geral, através da artéria hepática, e do sistema linfático. A capacidade de captação, especialmente de lipídios, pelos hepatócitos pode ser a principal explicação para o início da esteatose hepática não alcoólica ${ }^{25}$. $\mathrm{O}$ aumento na deposição de gordura no fígado pode ser também ocasionado devido ao aumento na disponibilidade de ácidos graxos livres ( $A G L)$, com concomitante ausência de aumento na $\beta$-oxidação ${ }^{26}$. Nesse sentido, os resultados reforçam esta teoria, pois os animais alimentados com dieta hiperlipídica apresentaram maior quantidade de gordura depositada no fígado, expressa em percentagem (\%), quando comparados com o grupo alimentado com dieta padrão (Figura 1). Entretanto, o peso relativo do fígado não apresentou alteração (Tabela 2) podendo ter ocorrido devido à diminuição do conteúdo de glicogênio nesse tecido, em resposta à dieta hiperlipídica ${ }^{26}$.

Quanto ao perfil lipídico, o consumo excessivo de gordura está relacionado com o desenvolvimento de dislipidemias ${ }^{20,26-29}$, como observado neste estudo, uma vez que a dieta hiperlipídica aumentou as concentrações séricas de TG, colesterol total e lipoproteína de alta densidade (Figura 2). Esses resultados reforçam o papel da dieta hiperlipídica como modelo experimental de desenvolvimento de obesidade e de síndrome metabólica. Todavia, a principal questão a ser respondida é: qual forma de intervenção é mais eficiente para reverter os efeitos causados pelo consumo de dieta hiperlipídica?

Dentre as formas de intervenção que visam a reduzir os efeitos adversos da dieta hiperlipídica e do sedentarismo, está a associação de uma dieta balanceada à prática regular de atividade física, que tem sido amplamente estudada11,12,22. Entretanto, as adaptações metabólicas dependem da intensidade, da freqüência e da duração do exercício ${ }^{30,31}$.

Diversos estudos experimentais reportam que o Exercício Moderado (90 minutos) promove redução no peso corporal e na adiposidade, além de melhorar o perfil lipídico $113,20,21,26$. Por outro lado, o conceito de acúmulo de atividade física, que consiste na prática de atividades físicas com duração mínima de 10 minutos por sessão, vem sendo amplamente divulgado ${ }^{9}$. Com relação à

Tabela 2. Peso relativo ( $\mathrm{g}$ de tecido/100g de peso corporal) dos tecidos adiposos brancos epididimal, retroperitoneal e visceral e fígado de grupos experimentais (ratos Wistar). São Carlos (SP), 2007.

\begin{tabular}{|c|c|c|c|c|c|c|c|c|c|}
\hline \multirow{3}{*}{\multicolumn{2}{|c|}{ Grupos }} & \multirow{2}{*}{\multicolumn{2}{|c|}{$\begin{array}{c}\text { Adiposidade visceral } \\
\text { EPI }\end{array}$}} & \multicolumn{4}{|c|}{ Adiposidade central } & & \\
\hline & & & & \multicolumn{2}{|c|}{ VIS } & \multicolumn{2}{|c|}{ RET } & \multicolumn{2}{|c|}{ FIG } \\
\hline & & $M$ & DP & M & DP & $M$ & $\mathrm{DP}$ & $M$ & DP \\
\hline \multirow{2}{*}{ 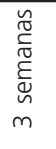 } & $P$ & 0,71 & 0,18 & 1,09 & 0,21 & 0,86 & 0,18 & 3,82 & 0,20 \\
\hline & $\mathrm{H}$ & 1,35 & $0,27^{*}$ & 1,92 & $0,34^{*}$ & 1,96 & $0,33^{*}$ & 3,82 & 0,22 \\
\hline \multirow{3}{*}{ 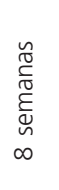 } & S & 1,14 & 0,42 & 1,03 & $0,24^{\circ}$ & 1,15 & $0,15^{\circ}$ & 3,36 & 0,24 \\
\hline & TC & 0,80 & $0,31^{\circ+}$ & 0,77 & $0,19^{\circ}$ & 0,76 & $0,30^{\circ}+$ & 3,50 & 0,24 \\
\hline & $\mathrm{Tl}$ & 0,67 & $0,19^{\circ+}$ & 0,81 & & 0,59 & $0,28^{\circ+}$ & 3,51 & 0,02 \\
\hline
\end{tabular}

Diferentes subscritos * versus $\mathrm{P} ;{ }^{\circ}$ versus $\mathrm{H}_{;}{ }^{+}$versus $\mathrm{S} ;{ }^{\#} \mathrm{TC} \times \mathrm{Tl} p<0,05$.

P: animais sedentários alimentados com dieta padrão durante 3 semanas; $\mathrm{H}$ : animais sedentários alimentados com dieta hiperlipídica durante 3 semanas; S: sedentário; TC: treinado contínuo; TI: treinado intermitente; M: média; DP: desvio-padrão; EPI: epididimal; RET: retroperitoneal; VIS: visceral. 
segunda fase deste estudo, a Tabela 1 demonstra que a utilização dos dois protocolos de treinamento (TC e TI) promoveu aumento na ingestão ener-

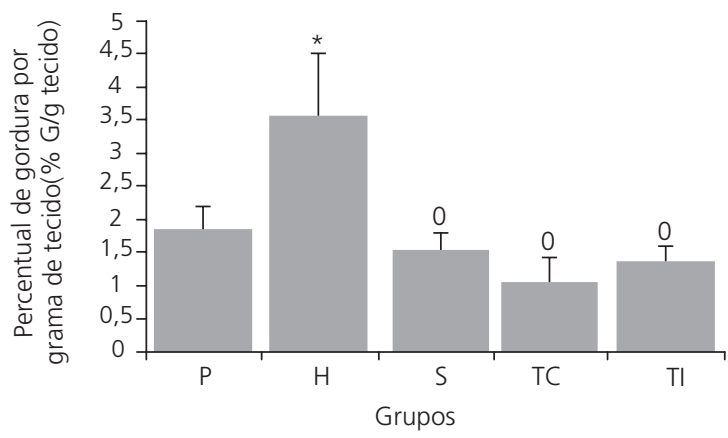

Figura 1. Percentual de gordura por grama de tecido (\%G/g tecido) do fígado de grupos experimentais (ratos Wistar). São Carlos (SP), 2007.

Nota: Os valores estão expressos como média (M) e desvio-padrão (DP).

Diferentes subscritos * versus $\mathrm{P} ;{ }^{0}$ versus $\mathrm{H}$.

$\mathrm{P}$ : animais sedentários alimentados com dieta padrão durante 3 semanas; $\mathrm{H}$ : animais sedentários alimentados com dieta hiperlipídica durante 3 semanas; S: sedentário; TC: treinado contínuo; TI: treinado intermitente.

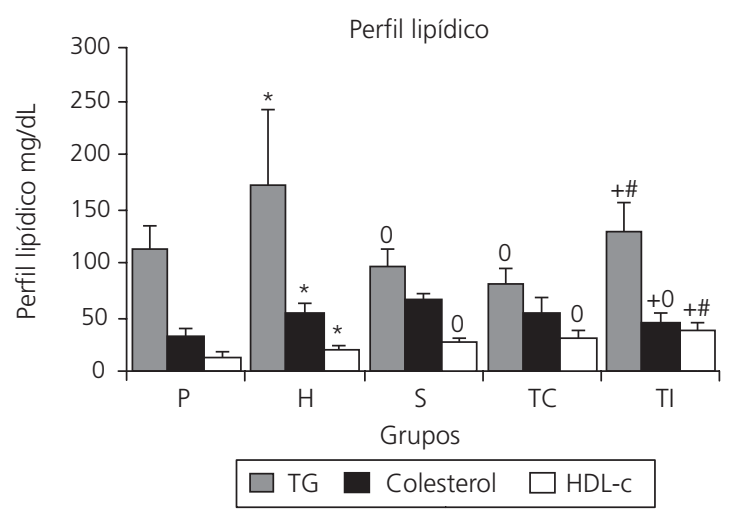

Figura 2. Concentração sérica ( $\mathrm{mg} / \mathrm{dL}$ ) de triacilglicerol, colesterol total e HDL-colesterol de grupos experimentais (ratos Wistar). São Carlos (SP), 2007.

Nota: Os valores estão expressos como média (M) e desvio-padrão (DP).

Diferentes subscritos ${ }^{*}$ versus $\mathrm{P} ;{ }^{0}$ versus $\mathrm{H} ;{ }^{+}$versus $\mathrm{S} ;$ \# $\mathrm{TC} \times \mathrm{TI}$ $p<0,05$.

P: animais sedentários alimentados com dieta padrão durante 3 semanas; $\mathrm{H}$ : animais sedentários alimentados com dieta hiperlipídica durante 3 semanas; S: sedentário; TC: treinado contínuo; TI: treinado intermitente; TG: triglicérides; HDL-c: lipoproteínas de alta densidade. gética (kcal) e no consumo de dieta (diário) comparado ao grupo sedentário. Apesar disso, somente os animais do grupo TI apresentaram um menor peso corporal final e, conseqüentemente, ganho de peso (\%), comparado aos dos grupos TC e $S$. Desse modo, pode-se sugerir que a mudança de dieta e os exercícios causaram modificação no metabolismo energético, sendo mais evidente no grupo que se exercitou de forma intermitente.

Sabe-se que, durante exercícios de 30 minutos de duração e de intensidade leve a moderada, o percentual de re-esterificação dos ácidos graxos livres reduz de $70 \%$ a, aproximadamente, $25 \%$. Essa diminuição, associada ao aumento (3 vezes) na liberação dos ácidos graxos livres advindos da hidrólise dos triacilgliceróis, resulta em um aumento de 6 vezes na disponibilidade de AGL para a oxidação. Além disso, o transporte dos AGL do tecido adiposo para o tecido muscular ativo, durante o exercício, também aumenta ${ }^{32}$. No presente estudo, essa adaptação ocorre três vezes por dia, durante o período de recuperação em resposta ao treinamento intermitente, suge-rindo um efeito pronunciado sobre o ganho de peso.

Tanto a mudança de dieta (S) quanto os treinamentos (TC e TI) foram capazes de promover redução na adiposidade central e visceral, quando comparados ao grupo $\mathrm{H}$, sendo esta redução mais evidente nos grupos treinados (Tabela 2). Além disso, os dois tipos de treinamento foram capazes de reduzir o percentual de gordura visceral em relação ao grupo obeso $(H)$ e ao S (Figura 3). A diminuição da gordura visceral é muito importante, uma vez que este tecido parece ser a ligação entre as muitas faces da síndrome metabólica como: intolerância à glicose, hipertensão arterial, dislipidemias, resistência insulínica e esteatose hepática não alcoólica ${ }^{2,11,12,22}$. Além disso, os tratamentos foram efetivos na redução do percentual de gordura no fígado comparados ao grupo $\mathrm{H}$ (Figura 1), suprimindo a possibilidade do desenvolvimento de esteatose hepática não alcoólica induzida pela dieta hiperlipídica26. 
A mudança de dieta (S) e o treinamento contínuo melhoraram o perfil lipídico dos animais, quando comparado com o grupo $\mathrm{H}$, pois promoveram diminuição dos TG e aumento do HDL-c (Figura 2). O exercício intermitente também aumentou a concentração da lipoproteína de alta densidade com relação aos grupos $\mathrm{H}$, S e TC e aumentou os TG circulantes, quando comparado aos grupos S e TC (Figura 2). Esse aumento nos TG circulantes pode estar relacionado ao tipo de treinamento, uma vez que a demanda energética neste caso é maior, sendo necessárias adaptações para a manutenção dos estoques de glicogênio para a próxima sessão de exercício ${ }^{21}$.

Em conclusão, o simples fato de trocar uma dieta hiperenergética por uma balanceada, foi capaz de reduzir o risco de desenvolvimento de obesidade e dislipidemias, pela diminuição da adiposidade e do percentual de gordura nos tecidos. Mas a melhor forma de intervenção para o controle da obesidade mostrou ser os dois tipos de exercícios associados à dieta balanceada, pois promoveram diminuição da adiposidade, do percentual de gordura no fígado e do tecido visceral

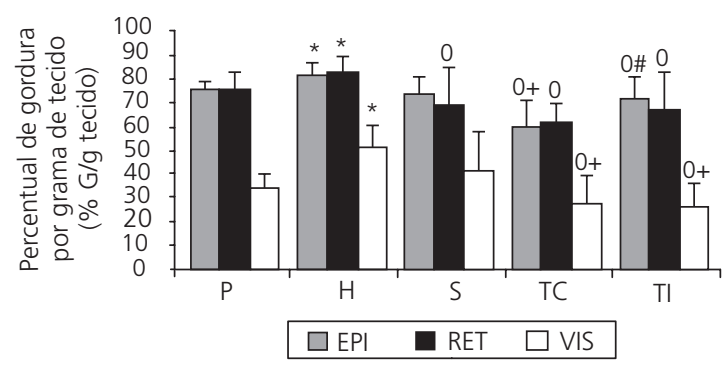

Figura 3. Percentual de gordura por grama de tecido (\% G/g tecido) dos tecidos adiposos brancos epididimal, retroperitoneal e visceral dos grupos experimentais (ratos Wistar). São Carlos (SP), 2007.

Nota: Os valores estão expressos como média (M) e desvio-padrão (DP).

Diferentes subscritos * versus $\mathrm{P} ;{ }^{0}$ versus $\mathrm{H} ;{ }^{+}$versus $\mathrm{S} ;{ }^{\#} \mathrm{TC} \times \mathrm{TI}$ $p<0,05$.

P: animais sedentários alimentados com dieta padrão durante 3 semanas; $\mathrm{H}$ : animais sedentários alimentados com dieta hiperlipídica durante 3 semanas; S: sedentário; TC: treinado contínuo; TI: treinado intermitente; EPI: epididimal; RET: retroperitoneal; VIS: visceral. e melhora no perfil lipídico. Portanto, os treinamentos aplicados neste estudo poderiam ser utilizados em modelo experimental e clínico, como estratégia no controle de peso e de dislipidemias, principalmente quando associado à dieta balanceada.

\section{COLABORADORES}

L. ZAMBON participou de todas as etapas do projeto de pesquisa. F.O. DUARTE colaborou com o desenvolvimento da pesquisa. L.F. FREITAS e F.R.R. SCARMAGNANI colaboraram com o projeto e a análise dos resultados. A. DÂMASO foi responsável pela apresentação dos métodos e das técnicas utilizadas na pesquisa. A.C.G.O. DUARTE co-orientou a pesquisa, como responsável pelo Laboratório de Nutrição e Metabolismo Aplicado ao Exercício e pelo grupo de pesquisa em Obesidade, Dislipidemias, Diabetes e Exercício, UFSCar. M. SENE-FIORESE orientou o projeto de pesquisa.

\section{REFERÊ NCIAS}

1. Dizdar O, Alyamaç E. Obesity: an endocrine tumor? Med Hypoteses. 2004; 63(5):790 2. doi:10.1016/j. mehy.2004.01.046.

2. Jequier E. Pathways to obesity. Int J Obes Relat Metab Disord. 2002; 26(2):S12-7. doi:10.1038/ sj.ijo.0802123.

3. Wajchenberg BL. Subcutaneous and visceral adipose tissue: their relation to the metabolic syndrome. Endocr Rev. 2000; 21(6):697-738.

4. Rosa EC, Zanella MT, Ribeiro AB, Kohlmann JO. Visceral obesity, hypertension and cardio-renal risk: a review. Arq Bras Endocrinol Metabol. 2005; 49(2): 196-204. doi: 10.1590/50004-27302005000 200005.

5. Westerterp KR. Perception passive overfeeding and energy metabolism. Physiol. Behav. 2006; 89(1): 62-5. doi:10.1016/j.physbeh.2005.12.014.

6. Saris WHM, Blair SN, van Baak MA, Eaton SB, Davies PS, Di Pietro L, et al. How much physical activity is enough to prevent unhealthy weight gain? Outcome of the IASO 1st Stock Conference and consensus statement. Obes Rev. 2003; 4(2):10114. doi:10.1046/j.1467-789X.2003.00101.x. 
7. Jakicic JM, Clark K, Coleman E, Donnelly JE, Foreyt J, Melanson E, et al. American College of Sports Medicine Position Stand. Appropriate intervention strategies for weight loss and prevention of weight regain for adults. Med Sci Sports Exerc. 2001; 33(12):2145-56

8. Pate RR, Pratt M, Blair SN, Haskell WL, Macera CA, Bouchard C, et al. Physical activity and public health. A recommendation from the Centers for Disease Control and Prevention and the American College of Sports Medicine. JAMA. 1995; 273(5):402-7.

9. Matsudo VKR. Exercícios acumulados funcionam? Diagn Tratamento. 2005; 10(3):163-5.

10. Brooks GA, Butte NF, Rand WM, Flatt JP, Caballero B. Chronicle of the Institute of Medicine physical activity recommendation: how a physical activity recommendation came to be among dietary recommendations. Am J Clin Nutr. 2004; 79(5): 921S-30S.

11. Duarte FO, Sene MO, Oishi J, Bidinotto $P$, Peres SEA, Moraes $G$, et al. O exercício de final de semana contribui para o controle das dislipidemias em ratos adultos machos alimentados com dieta rica em gorduras. Rev Bras Fisioter. 2003; 7(3):229-35.

12. Estadella D, Oyama LM, Dâmaso AR, Ribeiro EB, Oller do Nascimento CM. Effect of palatable hyperlipidic diet on lipid metabolism of sedentary and exercised rats. Nutrition. 2004; 20(2):218-24. doi:10.1016/j.nut.2003.10.008.

13. Burneiko RCM, Diniz YS, Galhardi CM, Rodrigues $H G$, Ebaid GM, Faine LA, et al. Interaction of hypercaloric diet and physical exercise on lipid profile, oxidative stress and antioxidant defenses. Food Chem Toxicol. 2006; 44(7):1167-72. doi: 10.1016/j.fct.2006.01.004.

14. Schnaider TB, Souza C. Aspectos éticos da experimentação animal. Rev Bras Anestesiol. 2003; 53(2):278-85.

15. Robinson AM, Willianson DH. Control of glucose metabolism in isolated acini of the lactating mammary gland of rat: effects of oleate on glugose utilization and lipogenesis. Biochem J. 1978; 170(3):609-13.

16. Manzoni MS, Rossi EA, Carlos IZ, Vendramini RC, Duarte AC, Dâmaso AR. Fermented soy product supplemented with isoflavones affected fat depots in juvenile rats. Nutrition. 2005; 21(10):1018-25. doi:10.1016/j.nut.2005.02.007.

17. Lovoie JM, Yasari S, Abdennadher M, Paquette A. Effects of alternations (10 days) of high-fat diet with normal diet on liver lipid infiltration, fat gain, and plasma metabolic profile in rat. Physiol Behav.
2005; 86(4):442-8. doi:10.1016/j.physbeh.2005. 07.018

18. Poobalan A, Aucott L, Smith WCS, Avenell A, Jung $\mathrm{R}$, Broom J, et al. Effects of weight loss in overweight/obese individuals and long-term lipid outcomes: a systematic review. Obs Rev. 2004; 5(1):43-50. doi: 10.1111/j.1467-789X.2004.00 127.x.

19. Hida K, Wada J, Eguchi J, Zhang H, Baba M, Seida A et al. Visceral adipose tissue-derived serine protease inhibitor: a unique insulin-sensitizing adipocytokine in obesity. Proc Natl Acad Sci USA. 2005; 102(30):10610-5. doi: 10.1073/pnas.0504 703102.

20. Schrauwen P, Westerterp KR. The role of high-fat diets and physical activity in the regulation of body weight. Br J Nutr. 2000; 84(4):417-27. doi:10.1017/ S0007114500001720.

21. Bernardes D, Manzoni MSJ, Souza CP, Tenório NM, Dâmaso AR. Effects of a high-fat diet and swimming moderated training on post exercise metabolism in male adults rat. Rev Bras Educ Fís Esp. 2004; 18(2):191-200.

22. Duarte ACGO, Fonseca DF, Manzoni MSJ, Soave CF, Sene-Fiorese M, Dâmaso AR, et al. High fat diet and secretory capacity of insulin in rat. Rev Nutr. 2006; 19(3):341-8. doi: 10.1590/S1415-5273 2006000300005.

23. Kretschmer BD, Schelling P, Beier N, Liebscher C, Treutel S, Krüger N, et al. Modulatory role of food, feeding regime and physical exercise on body weight and insulin resistance. Life Science. 2005; 76(14):1553-73. doi: 10.1016/j.Ifs.2004.08.028.

24. Gaíva MHG, Couto RC, Oyama LM, Couto GE, Silveira VL, Riberio EB, Nascimento CM. Polynsaturated fatty acid-rich diets: effect on adipose tissue metabolism in rats. Br J Nutr. 2001; 86(2):371-7.

25. Bradbury MW. Lipid metabolism and liver inflammation. I. Hepatic fatty acid uptake: possible role in steatosis. Am J Physiol Gastrointest Liver Physiol. 2006; 290(2):194-8. doi:10.1152/ajpgi. 00413.2005.

26. Gauthier MS, Couturier K, Latour JG, Lavoie JM. Concurrent exercise prevents high-fat-diet-induced macrovesicular hepatic steatosis. J Appl Physiol. 2003; 94(6):2127-34. doi:10.1152/japplphysiol. 01164.2002.

27. Diniz YS, Fernandes AAH, Campos KE, Mani F, Ribas BO, Novelli EL. Toxicity of hypercaloric diet and monosodium glutamate: oxidative stress and metabolic shifting in hepatic tissue. Food Chem 
Toxicol. 2004; 42(2):319-25. doi:10.1016/j.fct. 2003.09.006.

28. Iossa S, Lionetti L, Mollica MP, Crescenzo R, Botta M, Barletta A, et al. Effect of high-fat-feeding on metabolic efficiency and mitochondrial oxidative capacity in adults. Br J Nutr. 2003; 90(5):953-60. doi:10.1079/BJN2003000968.

29. Woods SC, Seeley RJ, Rushing PA, D’Alessio D, Tso P. A controlled high-fat diet induces an obese syndrome in rats. J Nutr. 2003; 133(4):1081-7.

30. Horowitz JF. Fatty acid mobilization from adipose tissue during exercise. Trends Endocrinol Metab. 2003; 14(8):386-92. doi:10.1016/S1043-2760 (03)00143-7.
31. Even PC, Rieth N, Roseau S, Laure-Achagiotis C. Substrate oxidation during exercise in the rat cannot fully account for training-induced changes in macronutrients selection. Metabolism. 1998; 47(7):777-82.doi:10.1016/S0026-0495(98) 90111-1.

32. Achten J, Jeukendrup AE. Optimizing fat oxidation through exercise and diet. Nutrition. 2004; 20(7-8): 716-27. doi:10.1016/j.nut.2004.04.005.

Recebido em: 28/8/2007

Versão final reapresentada em: 12/8/2008 Aprovado em: 9/2/2009 